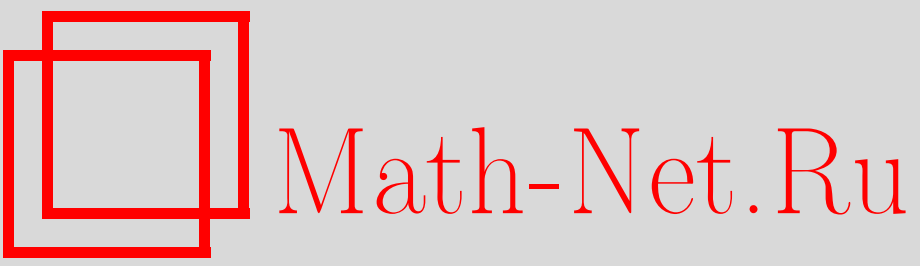

Я. Ю. Барт, Хеджирование опционов в биномиальной модели с разными процентными ставками, УМН, 1998, том 53, выпуск 5, 227-228

DOI: https://doi.org/10.4213/rm76

Использование Общероссийского математического портала Math-Net.Ru подразумевает, что вы прочитали и согласны с пользовательским соглашением

http://www.mathnet.ru/rus/agreement

Параметры загрузки:

IP : 54.196 .121 .252

26 апреля 2023 г., 13:37:23 


\title{
ХЕДЖИРОВАНИЕ ОПЦИОНОВ В БИНОМИАЛЬНОЙ МОДЕЛИ С РАЗНЫМИ ПРОЦЕНТНЫМИ СТАВКАМИ
}

\author{
Я. Ю. БАРТ
}

1. В работе [1] изложена методика хеджирования опционов для диффузионной модели финансового рынка с ограничениями. Задачей настоящей заметки является распространение методов указанной статьи на биномиальную модель финансового рынка с разными процентными ставками для описания полной структуры оптимального хеджа.

Рассмотрим следующую биномиальную модель финансового рынка (см. [3], [4]):

(1) $\quad \underline{B}_{n}=(1+\underline{r}) \underline{B}_{n-1}, \quad \bar{B}_{n}=(1+\bar{r}) \bar{B}_{n-1}, \quad S_{n}=\left(1+\rho_{n}(\omega)\right) S_{n-1}, \quad \underline{B}_{0}, \bar{B}_{0}, S_{0}>0$,

где $\underline{r} \leqslant \bar{r}$ - ставки депозита и кредита, $\rho=\left(\rho_{n}(\omega)\right)$ - последовательность н.о.р. случайных величин, принимающих значения $a$ и $b$ с вероятностью $1-p$ и $p$ соответственно $(a>b, 0<p<1)$. "Вероятность" $p$ порождает меру Р на подмножествах пространства $\Omega=\{+1,-1\}^{N}$.

Инвестиционной стратегией (портфелем) назовем предсказуемую последователшность $\pi=$ $\left(\underline{\beta}_{n}, \bar{\beta}_{n}, \gamma_{n}\right)$, где $\underline{\beta}_{n}, \bar{\beta}_{n}$ и $\gamma_{n}$ количества депозита, кредита и акций инвестора в момент времени $n$. Капитал стратегии $V_{n}^{\pi} \stackrel{\text { def }}{=} \underline{\beta}_{n} \underline{B}_{n}+\bar{\beta}_{n} \bar{B}_{n}+\underline{\gamma}_{n} S_{n}$, где $\underline{\beta} \geqslant 0, \bar{\beta} \leqslant 0$. Если капитал имеет вид: $V_{n}^{\pi}=V_{0}+\sum_{k=1}^{n}\left(\underline{\beta}_{k} \Delta \underline{B}_{k}+\bar{\beta}_{k} \Delta \bar{B}_{k}+\gamma_{k} \Delta S_{k}\right)-C_{n}$, где $\bar{C}=\left(C_{n}\right)_{0 \leqslant n \leqslant N}$ - возрастающая стохастическая последовательность, то $\pi$ называется стратегией с потреблением.

2. Для изложения важна характеризация стохастических последовательностей $V$ вида $V_{n}=$ $V_{0}+\sum_{k=1}^{n} H_{k}^{i} \Delta X_{k}^{i}-C_{n}$, где $H_{k}=\left(H_{k}^{1}, H_{k}^{2}, H_{k}^{3}\right)=\left(\underline{\beta}_{k}, \bar{\beta}_{k}, \gamma_{k}\right), X_{k}=\left(X_{k}^{1}, X_{k}^{2}, X_{k}^{3}\right)=$ $\left(\underline{B}_{k}, \bar{B}_{k}, S_{k}\right), C_{n}$ - возрастающая последовательность.

Пусть $\mathscr{Y}=\mathscr{Y}(H, X)=\left\{Y \mid Y=\sum H \Delta X\right\}, \mathscr{E}(\cdot)$ - "стохастическая экспонента" [1], [4].

Для $Y \in \mathscr{Y}$ имеет место разложение Дуба (см. [2]): $Y=M^{Y}+A^{Y}$, где $M^{Y}$ - мартингал, а $A^{Y}$ - предсказуемая последовательность (компенсатор $Y$ ). Семейство вероятностных мер Q таких, что $\mathrm{Q} \sim \mathrm{P}$ и $A^{\mathscr{Y}}(\mathrm{Q})_{n} \stackrel{\text { def }}{=} \operatorname{ess} \sup _{Y \in \mathscr{Y}} A_{n}^{Y}(\mathrm{Q})<+\infty, 0 \leqslant n \leqslant N$, где $A^{Y}(\mathrm{Q})$ - компенсатор $Y$ относительно меры $\mathrm{Q}$, обозначим $\mathscr{P}(\mathscr{Y})$.

Следующая "дуальная характеризация" (см. [1]) имеет ключевое значение.

Лемма. Предположим, ито $\mathscr{P}(\mathscr{Y}) \neq \varnothing$.

1) Неотричательная стохастическая последовательность $V$ представима в виде $V_{n}=V_{0} \mathscr{E}\left(\sum_{k=1}^{n} H_{k} \Delta X_{k}-C_{n}\right), 0 \leqslant n \leqslant N$, где $H$ - предсказуемая последовательность, $C$ - возрастающая последовательность, если и только если при всех $\mathrm{Q} \in \mathscr{P}(\mathscr{Y})$ процесс $V / \mathscr{E}\left(A^{\mathscr{Y}}(\mathrm{Q})\right)$ - супермартингал.

2) Для платежного обязательства $f_{N} c \sup _{\mathrm{Q} \in \mathscr{P}(\mathscr{Y})} \mathrm{E}^{\mathrm{Q}} f_{N} / \mathscr{E}\left(A^{\mathscr{Y}}(\mathrm{Q})\right)_{N}<+\infty$ найдется стохастическая последовательность $V$ такая, что

$$
V_{n}=\operatorname{essip}_{\mathrm{Q} \in \mathscr{P}(\mathscr{Y})}\left(\mathscr{E}\left(A^{\mathscr{Y}}(\mathrm{Q})\right)_{n} \mathrm{E}^{\mathrm{Q}}\left(f_{N} / \mathscr{E}\left(A^{\mathscr{Y}}(\mathrm{Q})\right)_{N} \mid \mathscr{F}_{n}\right)\right), \quad 0 \leqslant n \leqslant N,
$$

а для любой $\mathrm{Q} \in \mathscr{P}(\mathscr{Y})$ последовательность $V / \mathscr{E}\left(A^{\mathscr{Y}}(\mathrm{Q})\right)$ - супермартингал.

3. Обозначим $\mathscr{P}$ семейство вероятностных мер $\mathrm{Q}$ таких, что $\mathrm{Q} \sim \mathrm{P}$ и для всех $k, 1 \leqslant k \leqslant N$, вьполнено неравенство: $\underline{r} \leqslant \mathrm{E}^{\mathrm{Q}}\left(\rho_{k} \mid \mathscr{F}_{k-1}\right) \leqslant \bar{r}$.

Теорема. 1) Положительная стохастическая последовательность $\left(V_{n}\right)_{0 \leqslant n \leqslant N}$ есть капитал стратегии с потреблением если и только если для всякой $\mathrm{Q} \in \mathscr{P}$

$$
V_{n} / \prod_{k=1}^{n}\left(1+\mathrm{E}^{\mathrm{Q}}\left(\rho_{k} \mid \mathscr{F}_{k-1}\right)\right), \quad 0 \leqslant n \leqslant N,
$$

является супермартингалом. 
2) Для опциона европейского типа с платежнным обязательством $f_{N} \geqslant 0$ множество хеджирующих стратегий не пусто если и только если

$$
\sup _{\mathbf{Q} \in \mathscr{P}} \mathrm{E}^{\mathrm{Q}} f_{N} / \prod_{k=1}^{N}\left(1+\mathrm{E}^{\mathrm{Q}}\left(\rho_{k} \mid \mathscr{F}_{k-1}\right)\right)<\infty .
$$

Существует минимальная хеджирующая стратегия с капиталом

$$
\widehat{V}_{n}=\operatorname{ess}_{\mathbf{Q} \in \mathscr{P}} \mathrm{E}^{\mathrm{Q}}\left(f_{N} / \prod_{k=n+1}^{N}\left(1+\mathrm{E}^{\mathrm{Q}}\left(\rho_{k} \mid F_{k-1}\right)\right) \mid \mathscr{F}_{n}\right) .
$$

Для доказательства п. 1 теоремы показывается, что для $\mathscr{Y}=\mathscr{Y}(\mathscr{H}, R)=\left\{Y \mid Y=a_{1} \underline{r}+\right.$ $\left.a_{2} \bar{r}+a_{3} \rho_{k}, \sum_{i=1}^{3} a_{i} \leqslant 1, a_{1} \geqslant 0, a_{2} \leqslant 0\right\}$ семейства $\mathscr{P}$ и $\mathscr{P}(\mathscr{Y})$ совпадают, $A_{n}^{\mathscr{Y}}(\mathrm{Q})=$ $\sum_{k=1}^{n} \mathrm{E}^{\mathrm{Q}}\left(\rho_{k} \mid \mathscr{F}_{k-1}\right)$, где $\mathrm{Q} \in \mathscr{P}(\mathscr{Y})$, и применяется первая часть леммы. Доказательство п. 2 проводится в два шага: сначала из супермартингальности (2) находим, что (3) является нижней границей для капитала, а с помощью второй части леммы, что указанная граница реализуется в виде капитала стратегии с потреблением.

4. Изложенньй подход с применением "обратной индукции" (см. [4]) приводит для платежного обязательства $f_{N}=g\left(S_{N}\right)$ к следующему выражению капитала минимального хеджа: $\widehat{V}_{n}=\widehat{v}\left(S_{n}, n\right)$, где $\widehat{v}\left(S_{n}, n\right)$ - решение уравнения:

$$
\left\{\begin{array}{l}
\widehat{v}\left(S_{N}, N\right)=g\left(S_{N}\right), \\
\widehat{v}\left(S_{n-1}, n-1\right)=\max \left\{\widehat{v}\left(S_{n-1}(1+a), n\right) \frac{\bar{r}-b}{a-b}+\widehat{v}\left(S_{n-1}(1+b), n\right) \frac{a-\bar{r}}{a-b},\right. \\
\left.\widehat{v}\left(S_{n-1}(1+a), n\right) \frac{\underline{r}-b}{a-b}+\widehat{v}\left(S_{n-1}(1+b), n\right) \frac{a-\underline{r}}{a-b}\right\} .
\end{array}\right.
$$

Если платежное обязательство $f_{N}=\sup _{n \leqslant N} S_{n}-S_{N}$, то капитал минимальной хеджирующей стратегии выражается в виде: $\widehat{V}_{n}=S_{n} \widehat{v}\left(X_{n}, n\right)$, где $X_{n}=\sup _{k \leqslant n} S_{k} / S_{n}$, а $\widehat{v}\left(X_{n}, n\right)-$ решение уравнения:

$$
\left\{\begin{array}{l}
\widehat{v}\left(X_{N}, N\right)=X_{N}-1, \\
\widehat{v}\left(X_{n-1}, n-1\right)=\max \left\{\widehat{v}\left(\max \left\{\frac{X_{n-1}}{1+a}, 1\right\}, n\right) \frac{(1+a)(\bar{r}-b)}{a-b}\right. \\
\quad+\widehat{v}\left(\max \left\{\frac{X_{n-1}}{1+b}, 1\right\}, n\right) \frac{(1+b)(a-\bar{r})}{a-b}, \widehat{v}\left(\max \left\{\frac{X_{n-1}}{1+a}, 1\right\}, n\right) \frac{(1+a)(\underline{r}-b)}{a-b} \\
\left.\quad+\widehat{v}\left(\max \left\{\frac{X_{n-1}}{1+b}, 1\right\}, n\right) \frac{(1+b)(a-\underline{r})}{a-b}\right\} .
\end{array}\right.
$$

Автор признателен Д. О. Крамкову и А. В. Мельникову за руководство данной работой.

\section{СПИСОК ЛИТЕРАТУРЫ}

[1] Волков С.Н., Крамков Д.О. // Обозрение прикл. и пром. матем. 1997. Т. 4. №1. С. 18-65. [2] Ширяев А.Н. Вероятность. М.: Наука, 1989. [3] Ширяев А. Н., Кабанов Ю. М., Крамков Д. О., Мельников А. В. // Теор. вероятн. и ее примен. 1994. Т. 39. № 1. С. 23-79. [4] Мельников А. В. Финансовые рынки. М.: ТВП, 1997. 